ARTICLE

https://doi.org/10.1038/s41467-019-08869-9

\title{
A platform for glycoengineering a polyvalent pneumococcal bioconjugate vaccine using $E$. coli as a host
}

\author{
Christian M. Harding ${ }^{1}$, Mohamed A. Nasr ${ }^{2,7}$, Nichollas E. Scott ${ }^{3}$, Guillaume Goyette-Desjardins (D) ${ }^{4}$, \\ Harald Nothaft ${ }^{2}$, Anne E. Mayer ${ }^{5}$, Sthefany M. Chavez ${ }^{5}$, Jeremy P. Huynh ${ }^{5}$, Rachel L. Kinsella ${ }^{5}$, \\ Christine M. Szymanski ${ }^{6}$, Christina L. Stallings ${ }^{5}$, Mariela Segura ${ }^{4} \&$ Mario F. Feldman ${ }^{1,5}$
}

Chemical synthesis of conjugate vaccines, consisting of a polysaccharide linked to a protein, can be technically challenging, and in vivo bacterial conjugations (bioconjugations) have emerged as manufacturing alternatives. Bioconjugation relies upon an oligosaccharyltransferase to attach polysaccharides to proteins, but currently employed enzymes are not suitable for the generation of conjugate vaccines when the polysaccharides contain glucose at the reducing end, which is the case for $~ 75 \%$ of Streptococcus pneumoniae capsules. Here, we use an O-linking oligosaccharyltransferase to generate a polyvalent pneumococcal bioconjugate vaccine with polysaccharides containing glucose at their reducing end. In addition, we show that different vaccine carrier proteins can be glycosylated using this system. Pneumococcal bioconjugates are immunogenic, protective and rapidly produced within $E$. coli using recombinant techniques. These proof-of-principle experiments establish a platform to overcome limitations of other conjugating enzymes enabling the development of bioconjugate vaccines for many important human and animal pathogens.

\footnotetext{
${ }^{1}$ VaxNewMo LLC, St. Louis, MO 63108, USA. ${ }^{2}$ Department of Biological Sciences, University of Alberta, Edmonton, AB T6G 2R3, Canada. ${ }^{3}$ Department of Microbiology and Immunology, Institute for Infection and Immunity, University of Melbourne at the Peter Doherty, Parkville, VIC 3010, Australia. ${ }^{4}$ Swine and Poultry Infectious Diseases Research Center, Faculty of Veterinary Medicine, University of Montreal, 3200 Sicotte Street, St-Hyacinthe, QC J2S 2M2, Canada. ${ }^{5}$ Department of Molecular Microbiology, Washington University School of Medicine, St Louis, MO 63110, USA. ${ }^{6}$ Department of Microbiology and Complex Carbohydrate Research Center, University of Georgia, Athens, GA 30602, USA. ${ }^{7}$ Present address: Department of Biology, Centre for Applied Synthetic Biology, Concordia University, Montreal, QC H4B 1R6, Canada. These authors contributed equally: Christian M. Harding, Mohamed A. Nasr. Correspondence and requests for materials should be addressed to C.M.H. (email: christian.harding@vaxnewmo.com) or to M.F.F. (email: mariofeldman@wustl.edu)
} 


\section{S} treptococcus pneumoniae (pneumococcus) is a leading cause of bacterial-induced pneumonia, meningitis, and bacteremia globally, particularly, afflicting children 5 years of age or younger ${ }^{1,2}$. Moreover, a 2000 epidemiological survey from the World Health Organization (WHO) estimated that 735,000 human immunodeficiency virus-uninfected children died from pneumococcal-related diseases ${ }^{2}$ with updated estimates slightly reduced to 541,000 deaths for the year 2008 (ref. ${ }^{3}$ ). An increase in the number of prophylactic treatment options, mainly due to advancements in pneumococcal vaccine developments, has emerged over the past two decades. Pneumovax ${ }^{\oplus} 23$, a 23 -valent polysaccharide vaccine, is used in elderly populations as well as children over the age of 2 years who are at increased risk of pneumococcal disease; ${ }^{4}$ however, polysaccharide vaccines typically act as $\mathrm{T}$ cell-independent antigens and are generally not effective in children 2 years of age and younger ${ }^{5}$. On the other hand, covalently linking a polysaccharide to a protein in the form of a conjugate vaccine elicits a $\mathrm{T}$ cell-dependent immune response across all age groups, characterized by high-affinity immunoglobulin G (IgG)-producing plasma cells and memory B cells $\mathrm{s}^{6,7}$.

Three pneumococcal conjugate vaccines have been commercially licensed since the year 2000: Prevnar ${ }^{\circledast}$, Synflorix ${ }^{\mathrm{T \omega}}$, and Prevnar $13^{\circledR}$. Prevnar $13^{\circledR}$, the most broadly protecting pneumococcal conjugate vaccine, is comprised of 13 proteinpolysaccharide conjugates consisting of pneumococcal serotypes $1,3,4,5,6 \mathrm{~A}, 6 \mathrm{~B}, 7 \mathrm{~F}, 9 \mathrm{~V}, 14,18 \mathrm{C}, 19 \mathrm{~A}, 19 \mathrm{~F}$, and 23F, each individually linked to the genetically inactivated diphtheria toxoid $\mathrm{CRM}_{197}$. Although highly protective in a three-dose schedule, Prevnar $13^{\circledast}$ is one of the most expensive vaccines on the market today. This is mainly due to its complex manufacturing process resulting in a cost of $\sim 600$ US dollars for primary and booster immunizations ${ }^{8}$. In fact, Prevnar $13^{\circledast}$ has been Pfizer's best-selling product for the fiscal years 2015-2017, with total revenues exceeding 17.5 billion US dollars ${ }^{9}$. Although pneumococcal conjugate vaccines have significantly reduced the burden of pneumococcal disease events ${ }^{10,11}$, due to variations in global serotype distributions ${ }^{12,13}$, serotype replacement events ${ }^{14}$, as well as the lack of a low-cost alternative for developing countries, alternative manufacturing strategies to expedite development of next generation vaccines are needed.

As mentioned above, currently licensed pneumococcal conjugate vaccines are synthesized chemically, which is a laborious process plagued with technical challenges, low yields, and batchto-batch variations ${ }^{15}$, highlighting the need for improved conjugate vaccine synthetic methodologies. Over the past 15 years, in vivo conjugation using bacterial protein glycosylation systems has emerged as a feasible alternative to chemical conjugation ${ }^{16}$, with multiple bioconjugate vaccine candidates now in various stages of development and clinical trials ${ }^{17,18}$. Bioconjugation is based on exploiting protein glycosylation, a ubiquitous posttranslational modification in which glycans are covalently linked to proteins. In bacteria, glycans are commonly bound to proteins via $\mathrm{N}$ - or $\mathrm{O}$-linkages on asparagine or serine/threonine residues, respectively ${ }^{19,20}$. Several pathways for bacterial glycosylation have been characterized, and among the best described are the oligosaccharyltransferase (OTase)-dependent pathways in Gramnegative bacteria $^{20}$. In these systems, a lipid-linked oligosaccharide is assembled sequentially at the cytoplasmic leaflet of the inner membrane, flipped to the periplasmic leaflet, and then transferred to acceptor proteins by either $\mathrm{N}$ - or O-OTases depending on the site of glycan attachment ${ }^{20}$. Many bacterial species, including $S$. pneumoniae, also synthesize capsular polysaccharides (CPSs) employing the same lipid-linked oligosaccharides prior to their polymerization, export, and transfer to the cell surface enabling their exploitation for bioconjugation reactions in Escherichia coli ${ }^{21}$.
Glycoproteins have been recombinantly synthesized in E. coli for use as vaccines ${ }^{16}$ and/or diagnostics 22,23 by co-expressing three components: a genetic cluster encoding for the proteins required to synthesize a glycan of interest, an OTase and an acceptor protein. One drawback of this process is the apparent glycan substrate specificity of the known OTases, which, for some of them, has been suggested to be dictated by the reducing end sugar ${ }^{24}$ (the first monosaccharide in the growing polysaccharide chain) of the lipidlinked oligo/polysaccharide of interest. Although OTases are able to transfer many different oligo- and polysaccharide structures ${ }^{24,25}$, some sugars have not been efficiently conjugated by known OTases to acceptor proteins. Therefore, characterizing different OTases is paramount for expanding our arsenal of therapeutic glycoproteins, including bioconjugate vaccines.

OTases currently used for commercially synthesizing glycoconjugates are the Campylobacter jejuni N-OTase $\mathrm{PglB}^{16}$ and the Neisseria meningitidis O-OTase PglL ${ }^{26}$, both of which exhibit a great deal of promiscuity towards glycan substrates ${ }^{24,25}$. However, neither enzyme has been experimentally demonstrated to conjugate glycans containing a glucose residue at the reducing end, such as $\sim 75 \%$ of $S$. pneumoniae CPSs ${ }^{19,27}$. In the present work, we demonstrate the first successful in vivo conjugation of $S$. pneumoniae CPSs containing glucose as the reducing end monosaccharide from multiple serotypes. This has been achieved using a different class of $O$-OTase, previously designated as $\mathrm{PglL}_{\mathrm{ComP}}$ by our group ${ }^{28}$, and henceforth termed PglS. Here, we present proof-of-concept studies on the engineering, characterization, and immunological responses of a polyvalent pneumococcal bioconjugate vaccine using the natural acceptor protein ComP as a vaccine carrier as well as a monovalent pneumococcal bioconjugate vaccine using a conventional vaccine carrier containing the Pseudomonas aeruginosa exotoxin A protein.

\section{Results}

PglS transfers pneumococcal CPS14 to its acceptor protein. PglB, the first OTase described, was shown to preferentially transfer glycans containing an acetamido group at the C-2 position of the reducing end sugar to asparagine residues of acceptor proteins ${ }^{24}$. However, polysaccharides with galactose $(\mathrm{Gal})$ at the reducing end, such as the Salmonella enterica Typhimurium O antigen, have been transferred by an engineered $\mathrm{PglB}$ variant ${ }^{29}$ and also by PglL, the $O$-OTase from Neisseria meningiditis ${ }^{25}$. However, there is no evidence available for PglB- or PglLmediated transfer of polysaccharides containing glucose (Glc) at the reducing end. We therefore tested the ability of PglB and PglL to transfer the pneumococcal CPS14, which has a Glc residue as the reducing end sugar, to their cognate acceptor proteins, AcrA and DsbA, respectively. As seen in Fig. 1a-f, no evidence for CPS14 glycosylation to either acceptor protein was observed.

Previously, we demonstrated that Acinetobacter species contain three O-linked OTases: a general PglL OTase responsible for glycosylating multiple proteins, and two pilin-specific OTases ${ }^{28}$. The first pilin-specific OTase is an ortholog of TfpO (also known as $\mathrm{PilO}$ ) and is not employed for in vivo conjugation systems due to its inability to transfer polysaccharides with more than one repeating unit ${ }^{26}$. The second pilin-specific OTase, PglS, glycosylates a single protein, the type IV pilin ComP ${ }^{28}$. A bioinformatic analysis indicated that PglS is the archetype of a distinct family of OTases, which prompted us to test its ability to transfer pneumococcal CPS14 to ComP. Western blotting analysis (Fig. 1g-i) showed that co-expression of the CPS14 biosynthetic locus in conjunction with PglS and a His-tagged variant of ComP resulted in a typical ladder-like pattern of bands compatible with protein glycosylation with multiple subunits. Both ComP-His (Fig. 1g) and CPS14 (Fig. 1h) were detected with antisera specific 
to each antigen; moreover, samples treated with proteinase $\mathrm{K}$ did not react with either the anti-His or anti-CPS14 antisera, indicating that the purified material is indeed proteinaceous. Together, these results suggest that, unlike the previously characterized OTases, PglS is able to transfer polysaccharides with Glc at the reducing end.

ComP is glycosylated at a serine residue in position $84 . \mathrm{N}$ glycosylation in bacteria generally occurs within the sequon D-X$\mathrm{N}-\mathrm{S}-\mathrm{T}$, where $\mathrm{X}$ is any amino acid but proline ${ }^{30}$. On the contrary, $O$-linked OTases do not seem to have defined recognition sequons. Most $O$-glycosylation events in bacterial proteins occur in regions of low complexity (LCR), rich in serine, alanine, and proline residues ${ }^{31,32}$. Some pilins are also $O$-glycosylated at a C-
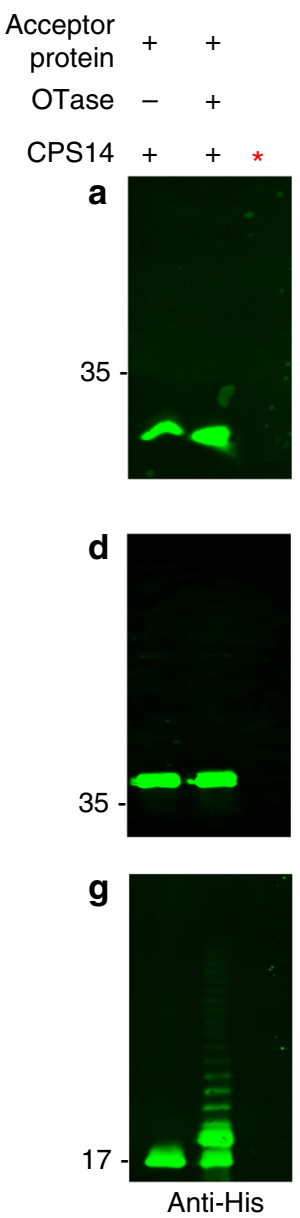
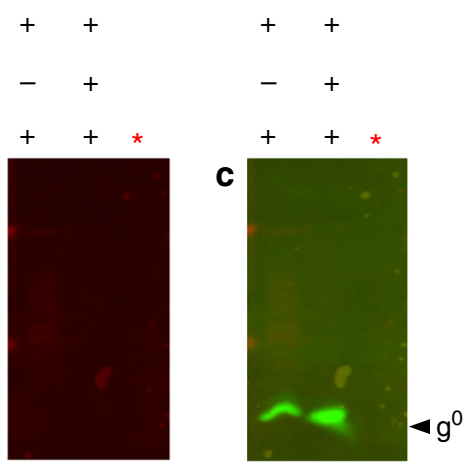

e

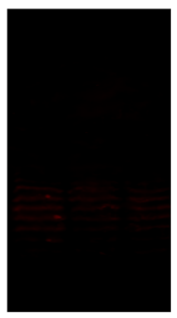

h

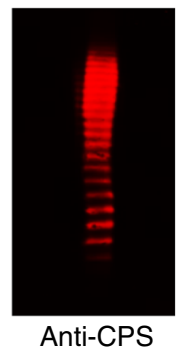

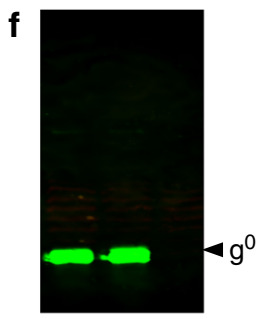

i

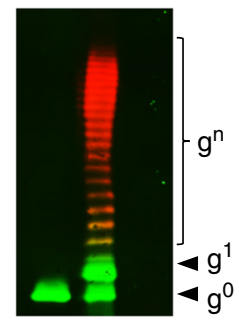

Merge
Fig. 1 PgIS can glycosylate the acceptor protein ComP with the pneumococcal CPS14 polysaccharide. Escherichia coli SDB1 cells coexpressing an acceptor protein (DsbA, AcrA, or ComP), an OTase (PglL, $P g \mid B$, or PgIS), and the CPS14 polysaccharide were analyzed for protein glycosylation via western blot analysis of the affinity-purified acceptor proteins. a-c DsbA purified from SDB1 cells in the presence or absence of PgIL. a Anti-His channel probing for Hexa-histidine tagged DsbA. b Antiglycan channel probing for CPS14. c Merged images for panels $a$ and $b$. d-f AcrA purified from SDB1 cells in the presence or absence of PglB. d Anti-His channel probing for Hexa-histidine tagged AcrA. e Anti-glycan channel probing for CPS14. f Merged images for panels $d$ and e. g-i ComP purified from SDB1 cells in the presence or absence of PgIS. $\mathbf{g}$ Anti-His channel probing for Hexa-histidine-tagged ComP. h Anti-glycan channel probing for CPS14. i Merged images for panels $g$ and $h$. The red asterisk indicates samples that were proteinase $\mathrm{K}$ treated for $1 \mathrm{~h}$ at $55^{\circ} \mathrm{C}$. Source data are provided as a Source Data file terminal serine residue ${ }^{33}$. We were unable to find an obvious LCR or a C-terminal serine residue in ComP homologous to those found in other pilin-like proteins and therefore employed mass spectrometry to determine the site(s) of glycosylation. Purified CPS14-ComP bioconjugates were subjected to GluC proteolytic digestion and multiple mass spectrometric analyses. As seen in Fig. 2, we identified a single glycopeptide consisting of a semiGluC-derived peptide ${ }_{81}$ ISASNATTNVATAT $_{94}$ attached to a glycan that matched the published CPS14 composition (Fig. 2a). To enable the confirmation of both the peptide and attached glycan sequences, multiple collision energies regimens were performed to confirm the glycosylation of the semi-GluC-derived peptide ${ }_{81}$ ISASNATTNVATAT $_{94}$ with a $1378.47 \mathrm{Da}$ glycan corresponding to $\mathrm{HexNAc}_{2} \mathrm{Hexose}_{6}$ (Fig. 2b). Additional glycopeptides were also observed decorated with extended glycans corresponding to up to four tetrasaccharide repeat units (Supplemental Fig. 1).

We have previously shown that Acinetobacter species predominantly glycosylate proteins at serine residues and thus hypothesized that either serine 82 or 84 was the site of glycosylation ${ }^{32}$. To determine which serine residue was the site of glycan attachment, we employed the C. jejuni heptasaccharide as the donor glycan, due to the ease at which glycosylation is detectable from whole-cell lysates. Wild-type ComP was glycosylated with the $C$. jejuni heptasaccharide as indicated by its increased electrophoretic mobility and signal co-localization with hR6 anti-glycan sera when co-expressed with PglS (Supplemental Fig. 2a-c). Mass spectrometry (MS) analysis also confirmed the presence of the $C$. jejuni heptasaccharide on the identical semiGluC-derived peptide ${ }_{81}$ ISASNATTNVATAT $_{94}$ modified by CPS14 (Supplemental Figs. 3 and 4). As a negative control, we generated a catalytically inactive PglS mutant (H324A), which when co-expressed with the $C$. jejuni heptasaccharide glycan was unable to glycosylate wild-type ComP (Supplemental Fig. 2A-C). We next performed site-directed mutagenesis and observed that glycosylation of ComP with the $C$. jejuni heptasaccharide was abolished in the ComP[S84A] mutant, whereas ComP[S82A] was glycosylated at wild-type levels (Supplemental Fig. 2A-C). In addition, the site of ComP glycosylation was also determined using a pneumococcal polysaccharide and is discussed below.

Immunogenicity of a monovalent CPS14-ComP bioconjugate. We evaluated the immunogenicity of a CPS14-ComP bioconjugate in a murine vaccination model. Two groups of mice $(n=10)$ individually received $3 \mu \mathrm{g}$ of either unglycosylated ComP or CPS14-ComP bioconjugate. Mice were boosted on days 14 and 28, and sacrificed on day 49 for whole-blood collection. Each vaccine was formulated based on total protein. Using an enzymelinked immunosorbent assay (ELISA) with a serotype 14 strain of S. pneumoniae adsorbed to each well, we compared IgM and IgG responses to CPS14. As seen in Supplemental Fig. 5, sera collected from mice vaccinated with a CPS14-ComP bioconjugate had an increased IgG response specific to CPS14 (Supp. Figure 5B), but not an increased IgM response (Supp. Figure 5A). Further, we employed secondary horseradish peroxidase (HRP)-tagged antiIgG subtype antibodies to determine which of the IgG subtypes were present in CPS14-ComP-vaccinated mice (Supp. Figure 5C). We determined that the CPS14-specific IgG1 response was higher than the other subtypes, which is consistent with previous findings for pneumococcal conjugate vaccines ${ }^{34,35}$.

Immunogenicity of a trivalent pneumococcal bioconjugate. There are more than 90 serotypes of S. pneumoniae 21,27 . Many increasingly prevalent serotypes, like serotypes $8,22 \mathrm{~F}$, and $33 \mathrm{~F}$, are not included in currently licensed vaccines ${ }^{36}$. Therefore, we 
a
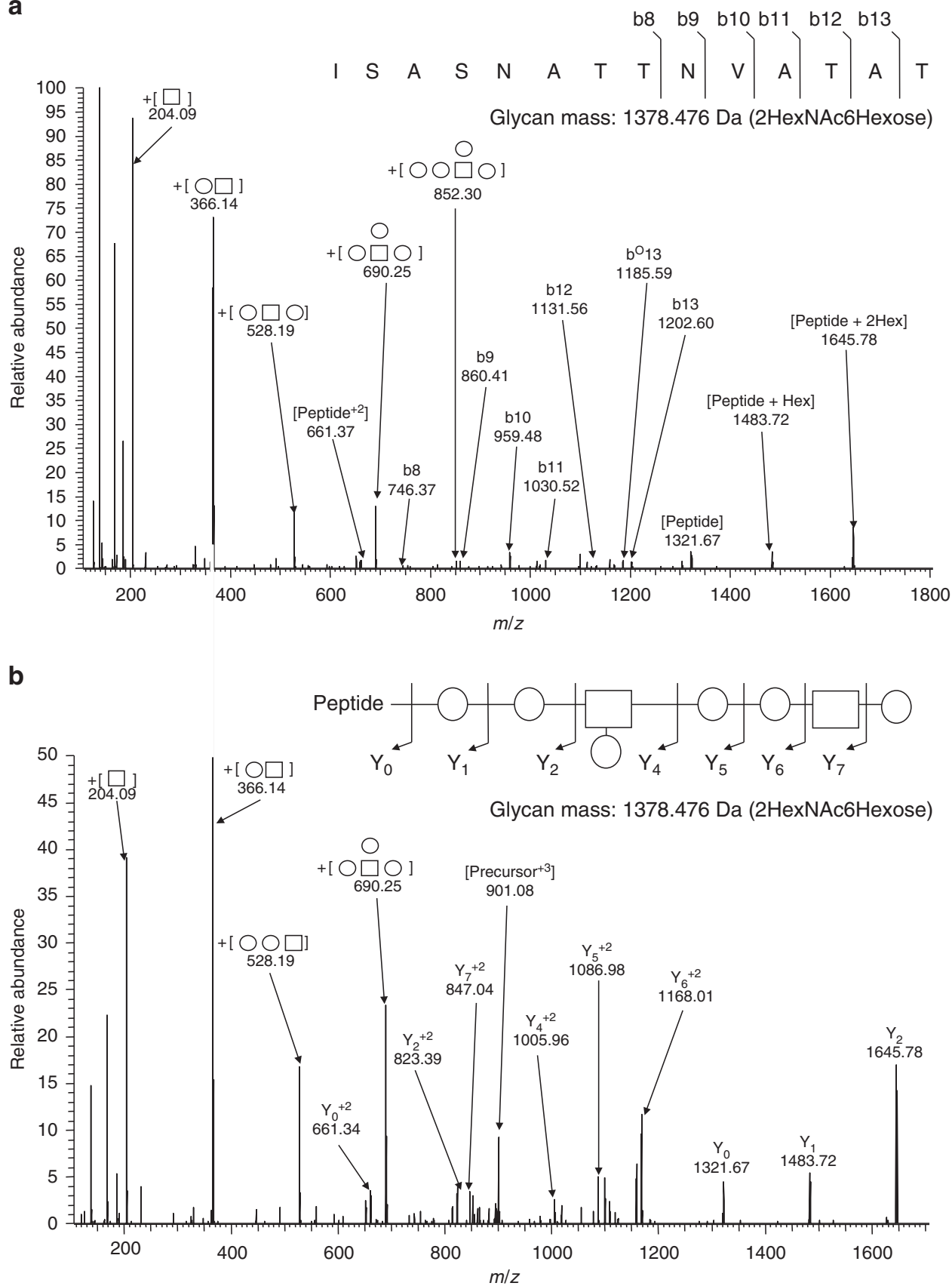

Fig. 2 Higher energy collisional dissociation (HCD) fragmentation spectra of GluC-digested CPS14-ComP bioconjugates. GluC-digested CPS14-ComP was subjected to HCD fragmentation enabling the confirmation of a single peptide attached to a glycan with the CPS14 repeating subunit. High collision energies (a) and low collision energies (b) regimens were undertaken to confirm the glycosylation of the peptide ${ }_{81}$ ISASNATTNVATAT 94 with a 1378.47 Da glycan corresponding to $\mathrm{HexNAc}_{2} \mathrm{Hexose}_{6}$

tested the versatility of PglS to generate a multivalent pneumococcal bioconjugate vaccine against two serotypes included in Prevnar $13^{\circledast}$ (serotypes $9 \mathrm{~V}$ and 14 ) and one serotype not included (serotype 8). The aforementioned CPSs all contain Glc as the reducing end sugar and are therefore not compatible with other commercially exploited conjugating enzymes. As seen in Figs. 3a-c and 3d-f, western blot analyses of affinity-purified proteins from whole cells co-expressing PglS, ComP, and either the CPS8 or CPS9V polysaccharides resulted in the generation CPS-specific ComP bioconjugates, respectively. Again, to confirm that the material purified was not contaminated with lipid-liked polysaccharides, we treated the samples with proteinase $\mathrm{K}$ and observed a loss of signal when analyzed via western blotting, confirming that the bioconjugates were proteinaceous.

Next, we performed a vaccination trial to determine the immunogenicity of a trivalent CPS8-ComP, CPS9V-ComP, and CPS14-ComP pneumococcal bioconjugate vaccine (Fig. 4a-l). Three control groups were included, one group receiving carrier protein alone (unglycosylated ComP), another group receiving a monovalent vaccine of the CPS14-ComP bioconjugate to account for IgG specificity when analyzing immune responses against other serotypes, and a third group receiving Prevnar $13^{\oplus}$ as a positive control. All immunogen groups contained an equal mixture of Freund's adjuvant, including mice receiving 


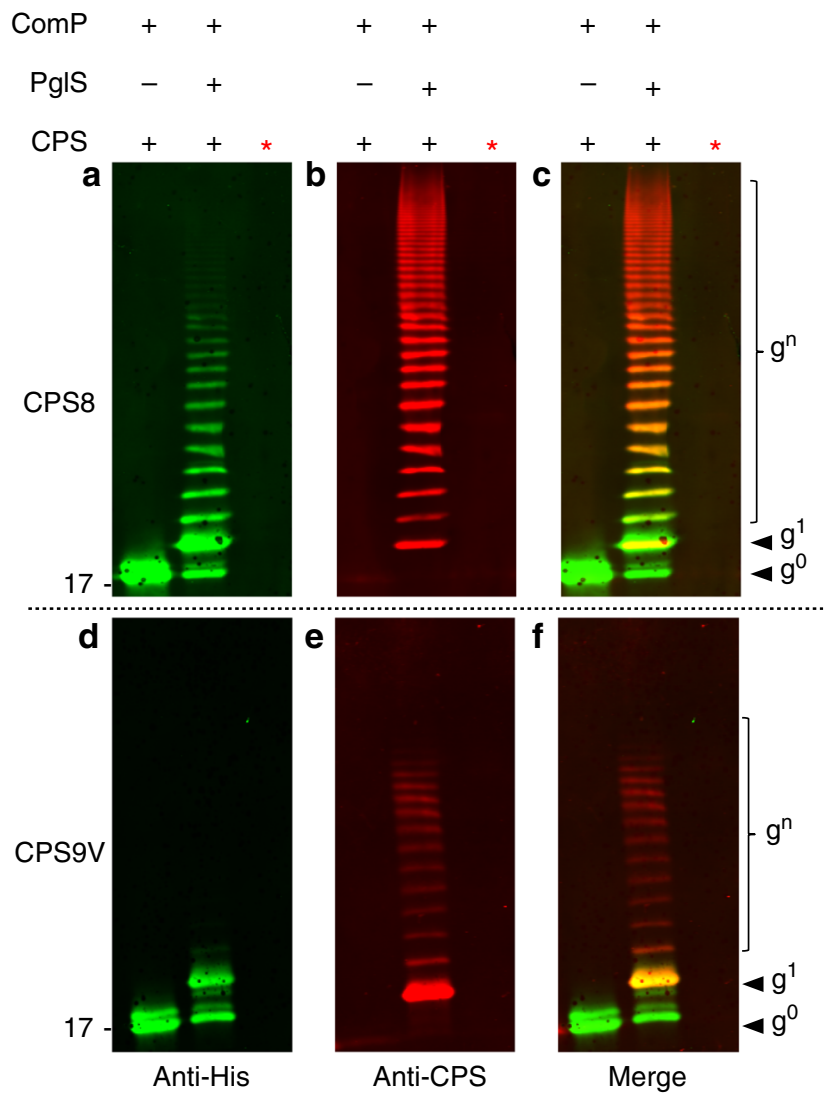

Fig. 3 Western blot analysis of CPS8-ComP and CPS9V-ComP glycoproteins. Escherichia coli SDB1 cells were prepared co-expressing ComP, PgIS, and either the pneumococcal CSP8 or CPS9V. Affinity-purified glycosylated ComP from each strain was analyzed for protein glycosylation via western blot analysis. a-c Western blot analysis of CPS8-ComP bioconjugates compared against ComP alone. a Anti-His channel probing for Hexa-histidine-tagged ComP purified from SDB1 expressing CPS8 in the presence or absence of PgIS. b Anti-glycan channel probing for CPS8. c Merged images for panels a and b. d-f Western blot analysis of CPS9VComP bioconjugates compared against ComP alone. d Anti-His channel probing for Hexa-histidine-tagged ComP purified from SDB1 expressing CPS9V in the presence or absence of PgIS. e Anti-glycan channel probing for CPS9V. f Merged images for panels $d$ and e. The red asterisk indicates samples that were proteinase $\mathrm{K}$ treated for $1 \mathrm{~h}$ at $55^{\circ} \mathrm{C}$. Source data are provided as a Source Data file

Prevnar $13^{\oplus}$. Day 49 sera from each group were analyzed by ELISAs on plates coated with S. pneumoniae serotypes 8, 9V, and 14. As mentioned above, serotypes $9 \mathrm{~V}$ and 14 are included in Prevnar $13^{\oplus}$ and an elevated IgG response could be seen in Prevnar $13^{\circledR}$-immunized mice against these two serotypes 49 days post vaccination. Mice receiving the monovalent CPS14-ComP bioconjugate also showed significant IgG increase specific to serotype 14 specific (Fig. 4i). Mice receiving the trivalent CPS8-/ CPS9V-/CPS14-ComP bioconjugate all had statistically significant increases in serotype-specific IgG responses 49 days post vaccinations (Fig. $4 \mathrm{j}-1$ ).

Because Freund's adjuvant is not a suitable adjuvant for human clinical development, we performed another immunization trial with vaccines formulated with Imject Alum Adjuvant, a mild adjuvant containing a mixture of aluminum hydroxide and magnesium hydroxide. Vaccination cohorts included a buffer/ adjuvant test group, a Prevnar $13^{\circledast}$ test group, and a trivalent CPS8-/CPS9V-/CPS14-ComP bioconjugate test group. Groups of three mice were vaccinated on days 1,14 , and 28 . Serum was collected on day 42 and used to determine effector functions via an opsonophagocytosis assay (OPA). Given the limited amounts of sera collected from individual mice, sera were tested for bactericidal activity against serotypes 8 and 14, as one serotype is included in Prevnar $13^{\circledR}$ (serotype 14) and one is not (serotype 8). As seen in Fig. 5a, b, serum from a representative mouse vaccinated with the trivalent CPS8-/CPS9V-/CPS14-ComP bioconjugate had increased bactericidal activity against $S$. pneumoniae serotype 14 strain when compared to sera from a mockvaccinated mouse. Importantly, that same bioconjugate vaccinated serum had high bactericidal activity against a $S$. pneumoniae serotype 8 strain, which was not observed for Prevnar $13^{\circledast}$-vaccinated sera due to the absence of this conjugate in its formulation.

Glycoengineering bioconjugates using a conventional carrier. $\mathrm{Up}$ to this point, we have exploited the use of ComP from Acinetobacter baylyi ADP1 as a carrier protein for pneumococcal bioconjugate vaccine production; however, we sought to increase the commercial applicability of this technology by engineering a conventional vaccine carrier to be compatible with our $O$-linked OTase. To this end, we generated a chimeric fusion protein consisting of the $\triangle \mathrm{E} 553$ variant of exotoxin A from P. aeruginosa (EPA) $\mathrm{C}$ terminally fused to a ComP fragment lacking its first 28 amino acids (ComP 428$)$. We used a ComP ortholog from Acinetobacter soli strain 110264 (accession number ENV58402) as it was most efficiently glycosylated by PglS and also found to be glycosylated at the same conserved serine as ComP from A. baylyi ADP1 (Supplemental Fig. 6). The EPA fusion was linked to ComP $\Delta 28$ with a glycine-glycine-glycine-serine linker and trafficked to the periplasm with a DsbA signal sequence.

Because current formulations of pneumococcal conjugate vaccines do not contain a conjugate for serotype 8 , we focused on generating an EPA-CPS8 pneumococcal bioconjugate. The EPA fusion was introduced into SDB1 cells co-expressing PglS and CPS8, subsequently purified, and then probed for glycosylation. As seen in Fig. 6a, b, the EPA fusion was efficiently glycosylated with CPS8 as determined by both western blot and Coomassie staining of purified glycoprotein. Furthermore, MS analysis of intact glycoproteins confirmed that the EPA fusion was repetitively modified with an increasing mass unit of $662 \mathrm{Da}$, which is the calculated mass of a single CPS8 subunit (Fig. 6c, d). The EPA fusion was found to be glycosylated with at least 11 CPS8 subunits by intact protein analysis; however, western blot and Coomassie analyses indicated that $>15$ subunits were able to be transferred.

Subsequently, we performed a vaccination experiment comparing the immunogenicity of an EPA-CPS8 pneumococcal bioconjugate to a ComP-CPS8 pneumococcal bioconjugate. Groups of 10 mice were either vaccinated with $5 \mu \mathrm{g}$ of EPA alone (based on total protein), $5 \mu \mathrm{g}$ of ComP-CPS8 (based on polysaccharide as determined by anthrone sulfuric acid), or 100 ng of EPA-CPS8 (based on polysaccharide as determined by MS of intact EPA-CPS8). Mice were vaccinated on days 1, 14, and 28 with serum collected on day 42 . All vaccines were formulated 1:1 with imject Alum Adjuvant. ELISAs were subsequently performed to determine the IgG titers specific to CPS8. As seen in Fig. 7a, mice vaccinated with either ComP-CPS8 or EPA-CPS8 had statistically significant increases in IgG titers specific to CPS8 when compared to EPA-vaccinated mice. Additionally, the protective capacity of sera from vaccinated mice was determined using a murine adapted OPA with whole-blood leukocytes. As shown in Fig. 7b, sera from vaccinated mice immunized with ComP-CPS8 displayed high levels of bactericidal killing ranging from 84 to $50 \%$, with one mouse not displaying any killing 
a

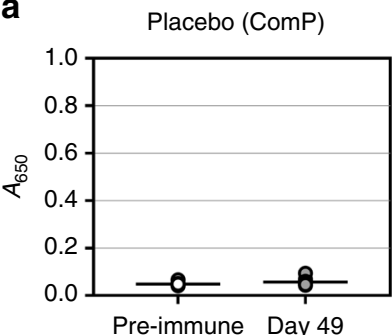

b
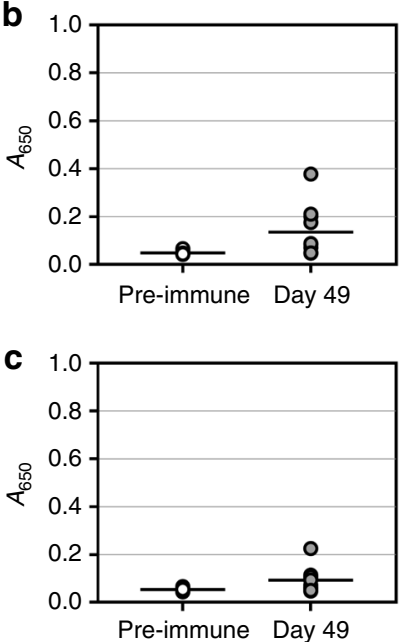

d

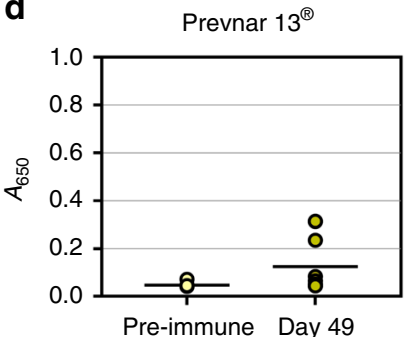

e
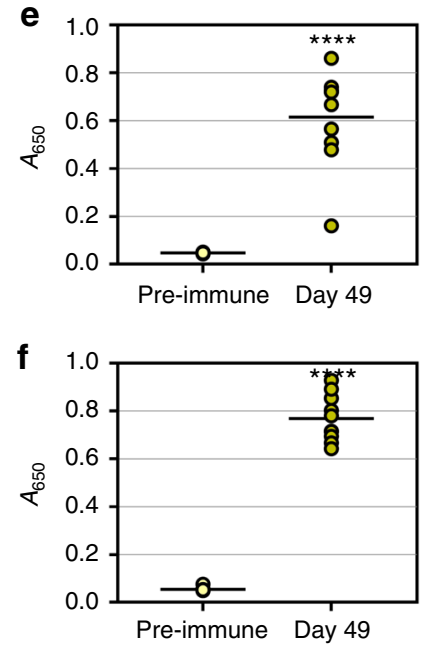

g
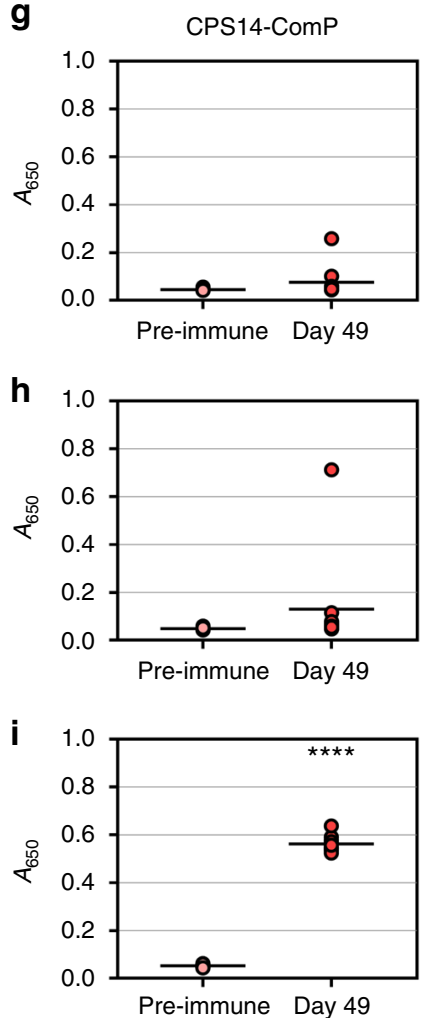

j
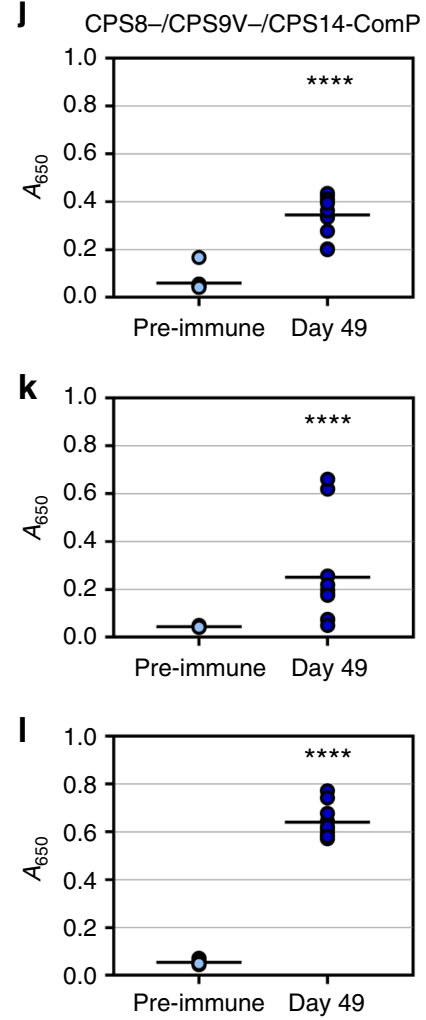

Fig. $4 \mathrm{Immunoglobulin} \mathrm{G}(\mathrm{IgG})$ responses of mice vaccinated with ComP, Prevnar $13^{\circ}$, a monovalent bioconjugate and a trivalent bioconjugate. Groups of mice were vaccinated with ComP alone, Prevnar $13^{\oplus}$, a monovalent CPS14-ComP bioconjugate vaccine, or a CPS8-/CPS9V-/CPS14-ComP biconjugate vaccine. Sera were collected on day 49 and analyzed for serotype-specific IgG responses via enzyme-linked immunosorbent assay (ELISA) compared against sera collected on day 0. a-c No lgG responses were detected in placebo vaccinated-mice for serotypes 8 (a), $9 \mathrm{~V}$ (b), or 14 (c). d-f Prevnar $13^{\oplus}-$ vaccinated mice did not have detectable IgG responses to serotype 8 (d), but did have lgG responses specific to serotype $9 \mathrm{~V}$ (e) and 14 (f). $\mathbf{g}$-i Mice vaccinated with a CPS14-ComP bioconjugate vaccine did not have IgG responses to serotypes 8 (g) or $9 \mathrm{~V}$ (h), but did have lgG responses to serotype 14 (i). j-I Trivalent CPS8-/CPS9V-/CPS14-ComP bioconjugate vaccinated mice all had statistically significant IgG responses to serotypes 8 (j), $9 \mathrm{~V}$ (k), and 14 (I). Unpaired $t$ tests (Mann-Whitney) were performed to statistically analyze pre-immune sera from day 49 sera. $P$ values for each case tested were ${ }^{\star \star \star \star} p=0.0001$. Each dot represents a single vaccinated mouse $(n=10$ mice per group). ELISA statistical calculations were performed on sera samples run in technical triplicates. Error bars indicate the standard deviation of the mean. Source data are provided as a Source Data file

activity. Moreover, sera from EPA-CPS8-vaccinated mice also displayed bactericidal ranging from 88 to $10 \%$, with three mice displaying no killing activity. Expectedly, sera from EPAvaccinated mice did not display killing activity.

\section{Discussion}

Traditional chemical conjugate vaccine synthesis is complex, costly, and laborious; ${ }^{15}$ therefore, new technologies to complement existing manufacturing pipelines are needed. One of these is bioconjugation, which has been thoroughly progressing as a feasible manufacturing alternative. The ability to glycosylate carrier proteins with polysaccharides containing Glc as the reducing end sugar has been elusive though, hindering the development of pneumococcal bioconjugate vaccines covering clinically relevant serotypes. Here we report the use of an $O$ linking OTase system for generating pneumococcal bioconjugate vaccines. Furthermore, we show that PglS naturally accepts polysaccharides containing Glc at the reducing end, a feat previously thought technically impossible due to substrate specificity limitations of all known conjugating enzymes.

The process of bioconjugation, described over a decade ago ${ }^{16}$, has proven to be both technically and commercially feasible. This is best evidenced by the 2015 partnership between GlaxoSmithKline and GlycoVaxyn for more than 200 million US dollars. To date, bioconjugation relies on two conjugating enzymes, PglB and PglL, with much of the focus on PglB due to its inherent ability to glycosylate soluble proteins at a known sequon ${ }^{30}$. As such, PglB has been the workhorse for the development and generation of bioconjugate vaccines in clinical trials. Examples include the Flexyn2a bioconjugate $^{18}$ against Shigella dysenteriae as well as a tetravalent ExPEc4V bioconjugate ${ }^{17}$ vaccine against extraintestinal pathogenic E. coli. Recently, PglB was used to generate a bioconjugate vaccine against serotype 4 of pneumococcus ${ }^{37}$. However, serotype 4 contains $N$-acetylgalactosamine as the reducing end sugar, which is one of the known substrates for PglB.

PglL also has commercially applicable features given its ability to transfer polysaccharides with $\mathrm{Gal}$ at the reducing end ${ }^{25}$. However, until recently, PglL was thought to only glycosylate a few Neisseria proteins ${ }^{31}$, most of which were membrane-associated proteins. Research by the Wang group though has resulted in the generation of a PglL-specific sequon that can be engineered onto any carrier protein and efficiently be glycosylated by PglL, thus rendering the production of PglL-manufactured bioconjugates more practical ${ }^{38,39}$. However, PglB and PglL are not useful for the production of the overwhelming majority pneumococcal serotypes due to the presence of Glc at their reducing ends.

The genome of $A$. baylyi ADP1 encodes for two O-OTases, a PglL ortholog, which is a general OTase, and PgIS, which glycosylates a single protein, ComP ${ }^{28}$. ComP is orthologous to type IV pilin proteins, like PilA from $P$. aeruginosa and PilE from 
a

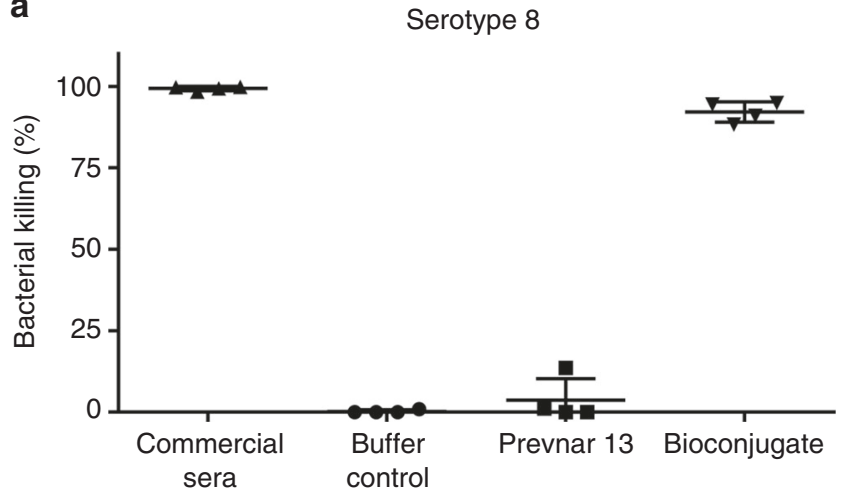

b

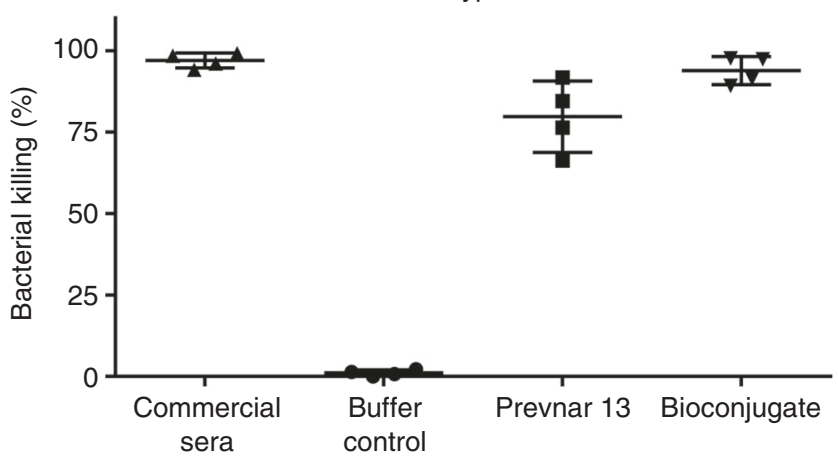

Fig. 5 Bactericidal activity of sera from vaccinated mice against Streptococcus pneumoniae serotypes 8 and 14. Opsonophagocytosis assays (OPAs) of sera from mice vaccinated with either buffer control ( $n=$ two female mice), Prevnar $13^{\circledast}$ ( $n=$ two female mice), or bioconjugate vaccine against both $S$. pneumoniae serotypes 8 (a) and 14 (b) ( $n=$ two female mice). OPAs were performed twice in order to have two biological replicates for interpretation. Serotype-specific commercial rabbit anti-S. pneumoniae sera were used as positive controls. A $5 \%\left(v^{-1}\right)$ sample serum and a bacterial multiplicity of infection (MOI) of 0.01 were added to fresh whole blood from naive mice to perform the assay. Viable bacterial counts were performed after $4 \mathrm{~h}$ of incubation. To determine bacterial killing, viable bacterial counts from tubes incubated with sample sera were compared to those incubated with control naive mouse sera. Results are expressed as percent bacterial killing for individual mice, with error bars representing the standard deviation of the mean. Source data are provided as a Source Data file

Neisseria gonorrhoeae, both of which are glycosylated by the OTases TfpO (also known as PilO) ${ }^{40}$ and PglL (also known as $\mathrm{PglO})^{41}$, respectively. TfpO glycosylates its cognate pilin at a Cterminal serine residue ${ }^{33}$, which is not present in ComP. Some Acinetobacter strains also possess TfpO orthologs ${ }^{28}$. PglL glycosylates PilE at an internal serine located at position 63 (ref. ${ }^{42}$ ). ComP contains serine residues near position 63 and the surrounding residues show limited conservation to PilE from $N$. gonnorrhoeae; however, Ser 63 and its surrounding residues were not part of the ComP glycosylation site. Instead, PglS glycosylates ComP at a single serine residue located at position 84, a glycosylation site which is not a canonical LCR, rich in proline, alanine, and serine residues. The ability of PglS to transfer polysaccharides containing Glc as the reducing end sugar coupled with the identification of a previously unrecognized site of glycosylation within the pilin superfamily demonstrates that PglS is a functionally distinct OTase from PglL and TfpO, and suggests that PglS belongs to a separate family of OTases.

Using the PglS/ComP OTase/acceptor protein pair, we have generated the first polyvalent pneumococcal bioconjugate vaccine and demonstrated its immunogenicity and efficacy using correlates of protection previously established as gold standards for pneumococcal conjugate vaccines ${ }^{43}$. First, we demonstrate serotype-specific IgG responses of CPS8-/CPS9V-/CPS14-ComPvaccinated mice. In these experiments, we found that the IgG response to all serotypes tested in bioconjugate-vaccinated mice were robust as determined by ELISA. Second, we showed that serum from a mouse vaccinated with pneumococcal bioconjugate vaccine was protective based on bactericidal killing assays against serotype 8 and 14 pneumococci. In addition, we have generated the first pneumococcal bioconjugate vaccine containing a conventional vaccine carrier. Namely, we have engineered the use of a ComP fragment as a glycotag, which can be added to the $\mathrm{C}$ terminus of EPA. We then paired the EPA fusion with the CPS8 polysaccharide and PglS, generating the EPA-CPS8 bioconjugate, a first of its kind pneumococcal bioconjugate vaccine. The EPACPS8 bioconjugate vaccine elicited high IgG titers specific to serotype 8 that were protective as determined via bactericidal killing. Importantly, vaccination with as little as $100 \mathrm{ng}$ of polysaccharide in the EPA-CPS8 bioconjugate was able to provide protection.

Even with the introduction and implementation of pneumococcal conjugate vaccines over the past two decades, hundreds of thousands of deaths are still attributed to pneumococcus each year ${ }^{10}$. This is due in part to the $90+$ serotypes of $S$. pneumoniae and the complex manufacturing methods required to synthesize pneumococcal conjugate vaccines. Together, these factors hinder development of broader, more protective and less costly variations of the vaccines. Our bioconjugation platform for synthesizing pneumococcal conjugate vaccines from polysaccharides with Glc at the reducing end could expedite development and lower manufacturing costs. PglS-derived bioconjugates could complement existing manufacturing pipelines or completely bypass the dependency on chemical conjugation methodologies, enabling the production of a more comprehensive pneumococcal conjugate vaccine. Here we present data using the natural acceptor protein, ComP, as well as a proof-of-principle EPA fusion protein as the targets of PglS glycosylation. However, future iterations of the EPA vaccine construct will impart additional sites of glycosylation to increase the glycan to protein ratio as well as expand upon the number of serotypes in order to develop a comprehensive pneumococcal bioconjugate vaccine. Regardless, we present compelling data indicating that these pneumococcal bioconjugates have the potential for further commercial development. Importantly, the platform technology we present in this study is not limited to pneumococcal polysaccharides, but in fact, has vast applicability for generating bioconjugate vaccines for many important human and animal pathogens that are incompatible with PglB and PglL. Notable examples include the human pathogens Klebsiella pneumoniae and Group B Streptococcus as well as the swine pathogen Streptococcus suis, all immensely relevant pathogens with no licensed vaccines available.

\section{Methods}

Bacterial strains, plasmids, and growth condition. Strains and plasmids used in this work are listed in Supplemental Table 1. Unless otherwise stated, E. coli strains were grown in Terrific Broth (TB) at $37^{\circ} \mathrm{C}$ overnight for ComP glycoprotein production or Super Optimal Broth (SOB) at $30^{\circ} \mathrm{C}$ overnight for EPA glycoprotein production. Streptococcus pneumoniae strains were grown in brain heart infusion (BHI) broth or sheep blood agar plates at $37^{\circ} \mathrm{C}$ in $5 \% \mathrm{CO}_{2}$. For plasmid selection, the antibiotics were used at the following concentrations: ampicillin $\left(100 \mu \mathrm{g} \mathrm{mL}^{-1}\right)$, tetracycline $\left(20 \mu \mathrm{g} \mathrm{mL}^{-1}\right)$, chloramphenicol

$\left(12.5 \mu \mathrm{g} \mathrm{mL}^{-1}\right)$, kanamycin $\left(20 \mu \mathrm{g} \mathrm{mL}^{-1}\right)$, and spectinomycin $\left(80 \mu \mathrm{g} \mathrm{mL}^{-1}\right)$ were added as needed. Oligonucleotides used in this study are listed in

Supplementary Table 2 . 
a

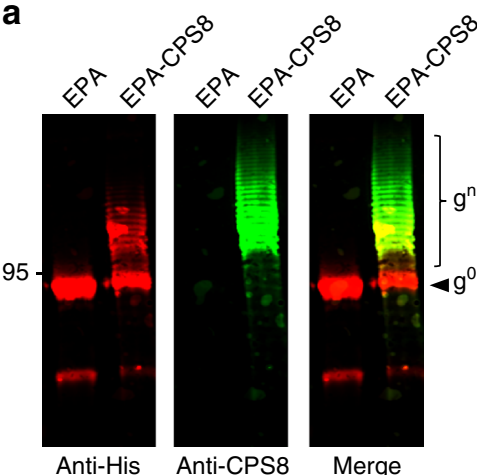

b

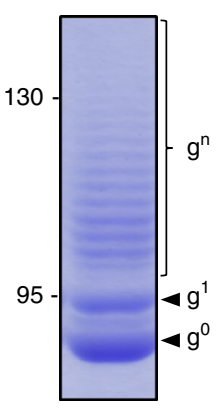

C

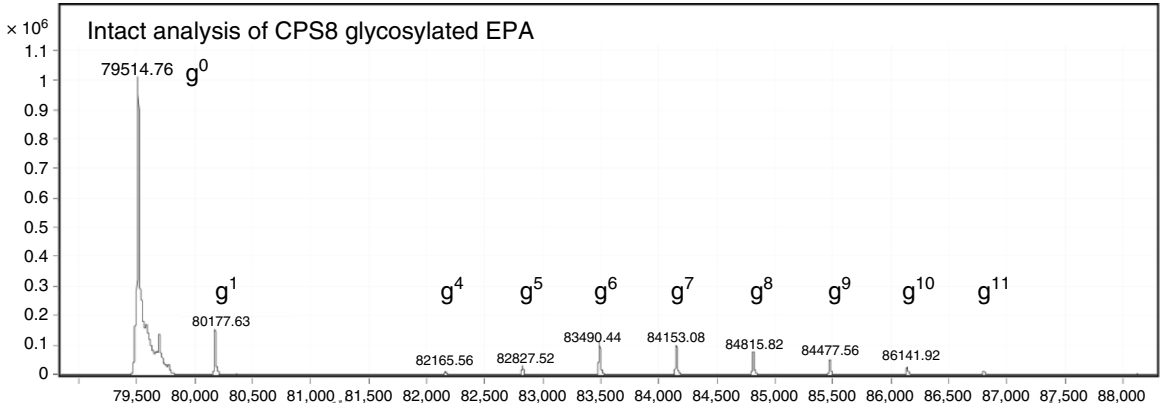

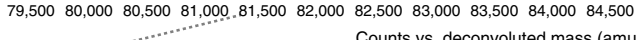

d

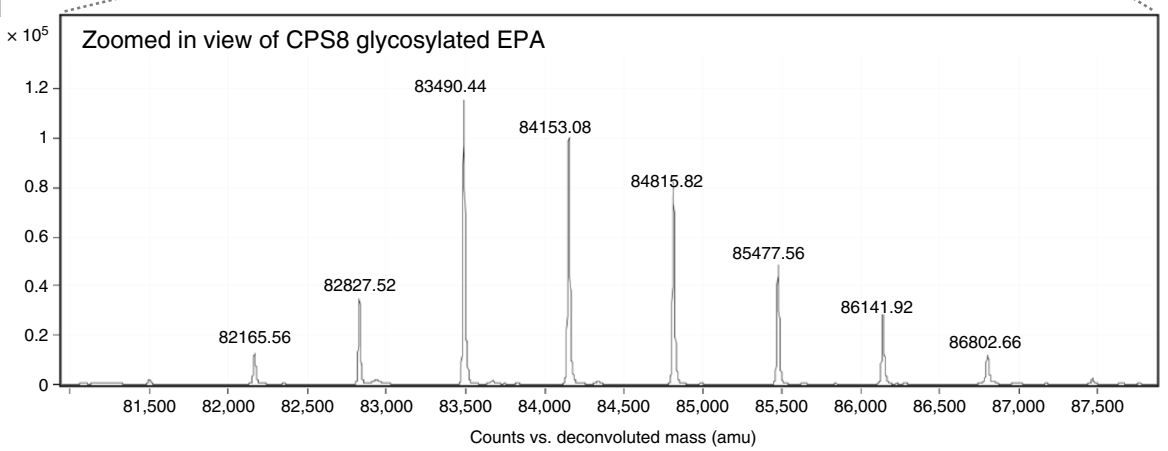

Fig. 6 Analysis of exotoxin A from Pseudomonas aeruginosa (EPA) glycosylation with the CPS8 capsular polysaccharide. Western blot analysis of EPA-CPS8 bioconjugates compared against EPA alone. a(Left panel Anti-His channel probing for Hexa-histidine-tagged EPA purified from SDB1 expressing CPS8 in the presence or absence of PgIS. a (Middle panel) Anti-glycan channel probing for CPS8. a (Right panel) Merged images for left and middle panels. $\mathbf{b}$ EPACPS8 separated on a SDS- polyacrylamide gel stained with Coomassie. c, $\mathbf{d}$ Intact protein mass spectrometry analysis showing the MS1 mass spectra for purified EPA-CPS8. The EPA fusion protein has a theoretical mass of 79,526.15Da and can be observed as the peak at 79,514.76 Da. The EPA fusion protein was also observed in multiple states of increasing mass corresponding to the CPS8 repeating subunit, which has a theoretical mass of 662Da. Varying glycoforms of the EPA-CPS8 were observed and are denoted by "gnumeric", where " $\mathrm{g}$ " stands for glycoform and the "numeric" corresponds to the number of repeating CPS8 subunits. The EPA fusion protein was modified with up to 11 repeating subunits of the CPS 8 glycan. Panel $d$ provides a zoomed in view of the varying EPA-CPS8 glycoforms. Source data are provided as a Source Data file

Heterologous glycosylation in E. coli. Escherichia coli SDB1 was made electrocompetent by growing cells to mid-logarithmic stage followed by two rounds of washing in $10 \%$ glycerol and a final resuspension in 1/250th of the original culture volume. Cells were electroporated with plasmids encoding the glycan synthesis loci, acceptor proteins, and OTases. Colonies were picked and grown at $37^{\circ} \mathrm{C}$ in TB or SOB with appropriate antibiotic selection and immediately induced with $0.05-0.1 \mathrm{mM}$ isopropyl $\beta$-D-1-thiogalactopyranoside or $0.2 \%$ arabinose as needed and left overnight at $37^{\circ} \mathrm{C}$. Cultures requiring arabinose induction received a second dose of arabinose after $4 \mathrm{~h}$. Cell pellets were obtained at stationary phases and prepared for western blot analysis.

Western blotting. Cell lysates containing the equivalent of $\mathrm{OD}_{600}=0.1$ units were loaded on $12.5 \%$ or $7 \%$ in-house prepared sodium dodecyl sulfate-polyacrylamide gel electrophoresis (SDS-PAGE) gels, which were then transferred to nitrocellulose membranes (Bio-Rad). Western blots were employed to determine protein modification. Primary antibodies included Pneumococcus Type 8 Serum (Ref. \# 16751), Pneumococcus Type 9 Serum (Ref. \# 16903), and Pneumococcus Type 14 Serum (Ref. \# 16751), all used at 1:1000 dilutions. Additional antibodies included 6x-His Tag Monoclonal Antibody (HIS.H8) (Catalog \# MA1-21315), used at 1:1000, and anti-Pseudomonas exotoxin A antibody (P2318-1ML), used at 1:5000. Secondary antibodies included Licor IRDye 680RD goat anti-mouse (925-68070) and goat anti-rabbit $800 \mathrm{CW}$ (926-32211) used at 1:10,000 dilutions. Western blotting was performed according to our previously published protocols ${ }^{28}$. Briefly, samples were separated by SDS-PAGE, transferred to nitrocellulose, blocked with Licor TBS blocking buffer, incubated with primary antibodies for $30 \mathrm{~min}$, washed three times in TBS supplemented with Tween-20, incubated with secondary antibodies for $30 \mathrm{~min}$, washed three times with TBS supplemented with Tween-20, and then visualized using an Odyssey Infrared Imaging System (LiCor Biosciences, USA).

Purification of proteins and glycoproteins. C terminally Hexa-histidine-tagged ComP and ComP bioconjugates were purified from E. coli total membrane preparations. Cells were grown overnight in $2 \mathrm{~L}$ of $\mathrm{TB}$ at $37^{\circ} \mathrm{C}$, washed with phosphate-buffered saline (PBS) buffer, and re-suspended in $60 \mathrm{~mL}$ of the same buffer. Cells were lysed by two rounds of cell disruption at approximately $20 \mathrm{kPSI}$ using a French press (Aminco), followed by the addition of a protease inhibitor cocktail (Roche). Lysates were centrifuged twice for $30 \mathrm{~min}$ at $20,000 \times g$ to pellet cell debris. Supernatants were ultra-centrifuged at $100,000 \times g$ for 60 mins to pellet total membranes. The pellets were re-suspended in PBS buffer containing $0.5 \%$ $n$-dodecyl- $\beta$-D-maltoside (DDM) and membrane proteins were solubilized by tumbling for $48 \mathrm{~h}$. An equal volume of PBS was added to the suspension to reduce detergent concentration to $0.25 \%$ and the suspension was ultra-centrifuged at $100,000 \times g$ for 60 mins. Solubilized membranes were filtered through 0.45 and $0.22 \mu \mathrm{m}$ filters and loaded on a His-Trap HP column (GE Healthcare) fitted to an ÄKTA purifier (Amersham Biosciences, Sweden). The column was equilibrated with a PBS/DDM buffer containing $20 \mathrm{mM}$ imidazole prior to loading the sample. Unbound proteins were removed by washing the column with seven column volumes of buffer containing 20 and $30 \mathrm{mM}$ imidazole in PBS stepwise. To elute proteins bound to the column, a gradient elution with an incremental increase in imidazole concentration was used. The majority of unconjugated and conjugated ComP eluted between 180 and $250 \mathrm{mM}$ imidazole. Imidazole was removed by an overnight round of dialysis followed by two 2 -h rounds through a $3.5 \mathrm{kDa}$ dialysis membrane (Spectrum labs) in a $250 \mathrm{~mL}$ dialysis buffer composed of PBS containing $0.25 \%\left(\mathrm{w} \mathrm{v}^{-1}\right)$ DDM. The final theoretical concentration of imidazole post dialysis was about $0.007 \mathrm{mM}$. Proteins were quantified using a DC kit (Bio-Rad), after which the samples were diluted to the appropriate concentrations for mouse immunizations.

C terminally Hexa-histidine-tagged EPA fusion proteins were purified from $E$. coli lysates lysed using mechanical disruption at 35,000 PSI using a cell disruptor from Constant Systems. Lysates were clarified at $15,000 \times g$ for $30 \mathrm{~min}$. The supernatents were passed over $3 \mathrm{~mL}$ of nickel NTA agarose, washed with 10 column volumes of buffer containing $20 \mathrm{mM}$ Tris, $10 \mathrm{mM}$ imidazole, $500 \mathrm{mM}$ $\mathrm{NaCl}, \mathrm{pH} 8.0$, and eluted with the same buffer containing $300 \mathrm{mM}$ imidazole. Eluted proteins were concentrated using an Amicon Ultra-15 concentrator, 
a

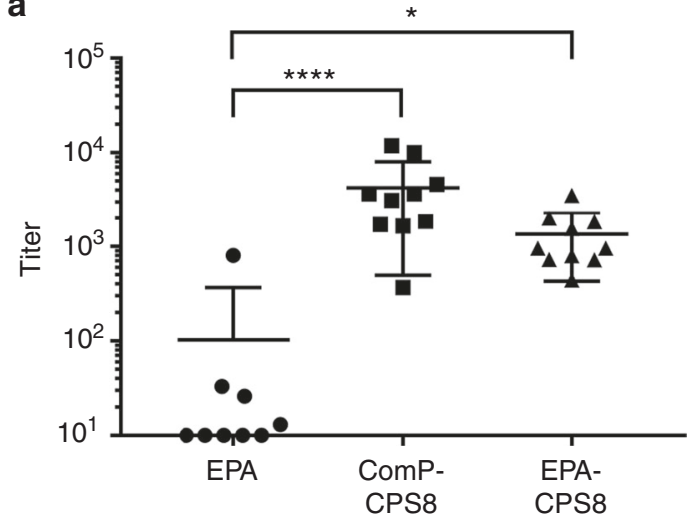

b

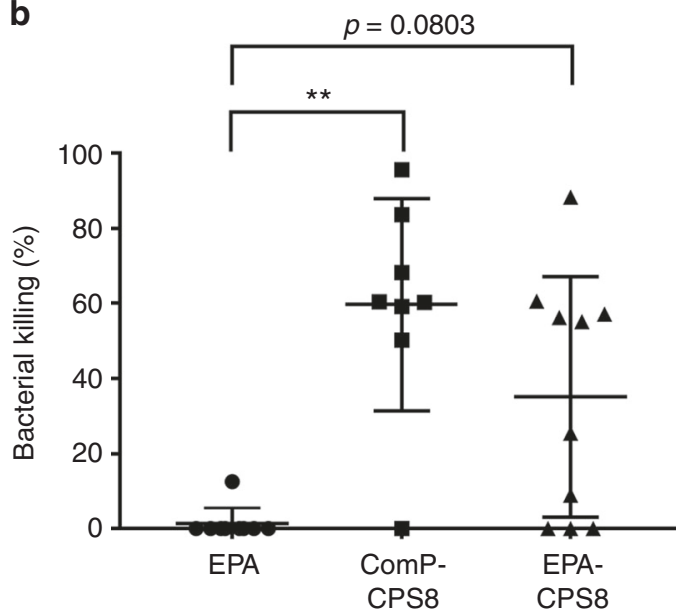

Fig. 7 Analysis of immune responses to ComP-CPS8 and EPA-CPS8 bioconjugates in mice. a Titers of CPS 8 IgG antibodies in mice immunized with CPS8 bioconjugate vaccines. Mouse groups were as follows: $\operatorname{EPA}(n=9$, mice vaccinated with $5 \mu \mathrm{g}$ of total protein), ComP-CPS 8 ( $n=10$, mice vaccinated with $5 \mu \mathrm{g}$ total polysaccharide), and EPA-CPS 8 ( $n=10$, mice vaccinated with $100 \mathrm{ng}$ of total polysaccharide). All mice were immunized with $100 \mu \mathrm{L}$ of a vaccine diluted 1:1 with Imject Alum Adjuvant on days 1, 14, and 28. Sera were collected on day 4. For the titration, enzyme-linked immunosorbent assay (ELISA) plates were coated with whole-cell serotype 8 pneumococci and incubated with 2-fold serial dilutions of sera. Each dot represents a single vaccinated mouse ( $n=10$ mice per group). ELISA statistical calculations were performed on sera samples run in technical triplicates. Titers for individual mice are shown, with error bars representing the standard error of the mean. Statistically significant titers compared to the EPA placebo group are denoted with asterisk and were determined using Kruskal-Wallis one-way analysis of variance (ANOVA). ${ }^{\star \star} P=0.0223$ and ${ }^{\star \star \star \star} P<0.0001$. For analysis and representation purposes, negative titer values $(<100)$ were given an arbitrary value of 10. b Opsonophagocytosis killing of $S$. pneumoniae serotype 8 by day 42 sera from mice immunized with ComPCPS8 and EPA-CPS8 bioconjugate vaccines. The same mouse groups described for the IgG titers were employed for the OPA. A 40\% $\left(v^{-1}\right)$ sample of serum and bacterial multiplicity of infection (MOI) of 0.01 were added to fresh whole blood from naive mice to perform the assay. Results are expressed as percent bacterial killing for individual mice, with error bars representing the standard deviation of the mean. Statistically significant killing compared to the EPA placebo group is denoted with asterisk and were determined using Kruskal-Wallis one-way ANOVA. ${ }^{\star \star} P=0.0015$. Source data are provided as a Source Data file

centrifuged at $10,000 \times g$ for $10 \mathrm{~min}$, and polished on a superdex 200 size exclusion column. Fractions enriched for EPA proteins pooled, concentrated, and buffer exchanged into PBS. Proteins were quantified using a DC kit for total protein concentrations and a modified anthrone sulfuric method for carbohydrate estimation. Briefly, to $100 \mu \mathrm{L}$ of sample, $4 \mathrm{~mL}$ of a $2 \mathrm{mg} \mathrm{mL}^{-1}$ anthrone- concentrated sulfuric acid solution was rapidly added. The sample was vortexed and heated in boiling water bath for $10 \mathrm{~min}$. Aliquots were measured for absorbance at $620 \mathrm{~nm}$. Carbohydrate estimations were based off a standard curve of the type 8 pneumococcal CPS from ATCC (cat. \# ATCC 20-X).

Murine model immunizations. All murine immunizations complied with all relevant ethical regulations for animal testing and research. Immunizations were conducted at the Southern Alberta Cancer Research Institute antibody services and Washington University School of Medicine in St. Louis according to institutional guidelines and received approval from the University of Calgary Animal Research and Education Executive Committee and the Institutional Animal Care and Use Committee at Washington University in St. Louis, respectively. For the CPS14ComP monovalent immunization, 4-6-week-old female BALB/c mice were injected with $50 \mu \mathrm{L}$ of purified protein/glycoprotein ( $3 \mu \mathrm{g}$ total protein) with $50 \mu \mathrm{L}$ of Freund's adjuvant. Two groups of mice $(n=10)$ were injected with either unglycosylated ComP (placebo) or CPS14-ComP bioconjugate. Sera from the mice were obtained before immunizations and $7,21,35$, and 49 days post immunizations. Booster doses were given on days 14 and 28. The same procedure was followed for the trivalent immunization, except four groups of mice $(n=10)$ were used for the four different immunization groups. These groups were injected with $100 \mu \mathrm{L}$ containing $3 \mu \mathrm{g}$ of unconjugated ComP (placebo) and Freund's adjuvant, $100 \mu \mathrm{L}$ containing $3 \mu \mathrm{g}$ of ComP-CPS14 conjugate and Freund's adjuvant, $100 \mu \mathrm{L}$ containing $9 \mu \mathrm{g}$ of a glycoprotein mixture (ComP-CPS8, ComP-CPS9V, and ComPCPS14) and Freund's adjuvant, or $100 \mu \mathrm{L}$ of a 1:10 diluted stock of Prevnar $13^{\circ}$ and Freund's adjuvant. CPS-ComP bioconjugates were formulated by total protein for this immunization.

Another trivalent immunization experiment was conducted with groups of three 4-6-week-old female BALB/c mice. Each immunization group was subcutaneously injected with $100 \mu \mathrm{L}$ of a 1:1 immunogen ( $3 \mu \mathrm{g}$ of protein of each of the trivalent bioconjugate or a 1:10 diluted stock of Prevnar $13^{\circ}$ ) to Imject Alum Adjuvant. Mice were vaccinated on days 0,14 , and 28 and then sacrificed on day 42 for sera collection.

A fourth immunization experiment was conducted with groups of three 4-6-week-old $\mathrm{BALB} / \mathrm{c}$ mice (five female and five male per group). Mice were immunized subcutaneously with $100 \mu \mathrm{L}$ of EPA ( $5 \mu \mathrm{g}$ total protein), $100 \mu \mathrm{L}$ of ComP-CPS8 ( $5 \mu \mathrm{g}$ total polysaccharide), or $100 \mu \mathrm{L}$ of EPA-CPS8 $(0.1 \mu \mathrm{g}$ total polysaccharide) on days 0,14 , and 28 and then sacrificed on day 42 for sera collection. Vaccines were formulated 1:1 with Imject Alum Adjuvant.

Enzyme-linked immunosorbent assays. Streptococcus pneumoniae strains grown overnight in BHI broth at $37^{\circ} \mathrm{C}$ in $5 \% \mathrm{CO}_{2}$ were washed in PBS and the optical density was adjusted to $\mathrm{OD}_{600}=0.6$ units. Cells were heat inactivated at $60^{\circ} \mathrm{C}$ for $2-4 \mathrm{~h}$ followed by immobilization on high binding 96 -well plates (Corning) by adding $50 \mu \mathrm{L}$ per well. Plates were incubated on a tumbler overnight at $4^{\circ} \mathrm{C}$. The following day, wells were washed three times with PBST (phosphate-buffered saline-Tween) $(100 \mu \mathrm{L}$ per well) before blocking with $5 \%$ skimmed milk $(250 \mu \mathrm{L}$ per well) for $2 \mathrm{~h}$. The wells were washed three times with PBST. Plates were incubated for $1 \mathrm{~h}$ at room temperature with mouse sera $(100 \mu \mathrm{L}$ per well) at a 1:500 dilution in $2.5 \%$ skimmed milk in PBST for assessing immunogenicity of ComP bioconjugates. For the positive control, commercial rabbit polyclonal antibodies against CPS were used (catalog numbers described above). Negative control wells were treated with skimmed milk without any primary antibody. After incubation with the primary antibody, wells were washed three times with PBST followed by a $1 \mathrm{~h}$ incubation with secondary HRP-conjugated anti-mouse IgG (Cat. \# 7076) diluted 1:4000 (100 $\mu \mathrm{L}$ per well) in $2.5 \%$ skimmed milk in PBST. After incubation, the wells were washed three times with PBST and $100 \mu \mathrm{L}$ of the chromogenic substrate TMB (Cell Signaling Technology) was added to each well. Plates were incubated at room temperature for $5 \mathrm{~min}$, after which the absorbance at $650 \mathrm{~nm}$ was measured using a BioTek ${ }^{\mathrm{m}}$ plate reader.

For IgG titer determinations, ELISA plates were coated with $100 \mu \mathrm{L}$ of $1 \times 10^{8}$ $\mathrm{CFU} \mathrm{mL} \mathrm{m}^{-1}$ of $S$. pneumoniae serotype 8 grown approximately to mid-log phase. Bacteria were washed twice in PBS and suspended in water prior to coating. ELISA plates were allowed to air dry in a biological hood for $24 \mathrm{~h}$. Fifty microliters of methanol were then added to each well and allowed to air dry. Plates were stored in a re-sealable bag protected from the light until use. To perform the titration of mouse total IgG antibodies, day 42 sera was serially diluted (2-fold) in PBST and antibodies were detected using an anti-mouse, HRP-linked IgG (Cell Signaling Technology \# 7076) diluted 1:4000. For mouse serum titrations, the reciprocal of the last serum dilution that resulted in an optical density at $450 \mathrm{~nm}$ equal to or lower than 0.2 was considered the titer of that serum. For representation purposes, negative titers (less than or equal to the cutoff) were given an arbitrary titer value of 10. Inter-plate variations were controlled by including an internal reference positive control on each plate. This control was hyper-immune sera from a mouse previously immunized with the ComP-CPS8 bioconjugate vaccine. The ELISA reactions in TMB were stopped when an OD450nm of $\sim 1$ was obtained for the internal positive control.

Site-directed mutagenesis. Mutagenic primers were designed using Primer X, a web-based primer design program (http://www.bioinformatics.org/primerx/). 
Primers used are listed in Supplemental Table 2. PCR reactions were performed using $P f u$ polymerase and $2-10 \mathrm{ng}$ of $\mathrm{pMN} 2$ as template. The PCR reaction consisted of an initial denaturation of $30 \mathrm{~s}$ at $95^{\circ} \mathrm{C}$ followed by 16 cycles of $30 \mathrm{~s}$ at $95^{\circ} \mathrm{C}, 60 \mathrm{~s}$ at $55^{\circ} \mathrm{C}, 360 \mathrm{~s}$ at $68^{\circ} \mathrm{C}$ with no final extension. PCR reactions were $D p n \mathrm{I}$ digested for $2 \mathrm{~h}$ to remove the template plasmid, then transformed into electrocompetent DH5a cells, and grown on ampicillin for plasmid selection. Colonies were sequenced to confirm mutagenesis.

Digestion of ComP-CPS14 conjugate. CPS14-ComP was affinity purified and separated via SDS-PAGE and Coomassie stained. SDS-PAGE separated CPS14ComP bands were excised and destained in a 50:50 solution of $50 \mathrm{mM}$

$\mathrm{NH}_{4} \mathrm{HCO}_{3}: 100 \%$ ethanol for $20 \mathrm{~min}$ at room temperature with shaking at $750 \mathrm{rpm}$. Destained bands were then washed with $100 \%$ ethanol, vacuum dried for $20 \mathrm{~min}$, and rehydrated in $10 \mathrm{mM}$ dithiothreitol (DTT) in $50 \mathrm{mM} \mathrm{NH}_{4} \mathrm{HCO}_{3}$. Reduction was carried out for $60 \mathrm{~min}$ at $56^{\circ} \mathrm{C}$ with shaking. The reducing buffer was then removed and the gel bands washed twice in $100 \%$ ethanol for $10 \mathrm{~min}$ to ensure the removal of remaining DTT. Reduced ethanol washed samples were sequentially alkylated with $55 \mathrm{mM}$ iodoacetamide in $50 \mathrm{mM} \mathrm{NH}_{4} \mathrm{HCO}_{3}$ in the dark for $45 \mathrm{~min}$ at room temperature. Alkylated samples were then washed with two rounds of Milli-Q water and $100 \%$ ethanol then vacuum dried. Alkylated samples were then

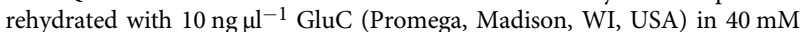
$\mathrm{NH}_{4} \mathrm{HCO}_{3}$ at $4^{\circ} \mathrm{C}$ for $1 \mathrm{~h}$. Excess GluC was removed, gel pieces were covered in $40 \mathrm{mM} \mathrm{NH}_{4} \mathrm{HCO}_{3}$, and incubated for $24 \mathrm{~h}$ at $37^{\circ} \mathrm{C}$. Peptides were concentrated and desalted using $\mathrm{C}_{18}$ stage tips $\mathrm{s}^{44,45}$ and stored on tip at $4{ }^{\circ} \mathrm{C}$. Peptides were eluted in Buffer B $(0.5 \%$ acetic acid, $80 \% \mathrm{MeCN})$ and dried before analysis by liquid chromatography-mass spectrometry (LC-MS).

Reversed phase LC-MS and HCD MS-MS. Purified peptides were re-suspended in Buffer $\mathrm{A}^{\star}$ and separated using an in-house packaged $25 \mathrm{~cm}, 75 \mu \mathrm{m}$ inner diameter, $360 \mu \mathrm{m}$ outer diameter, $1.7 \mu \mathrm{m} 130 \AA \mathrm{CSH} \mathrm{C}_{18}$ (Waters, Manchester, UK) reverse-phase analytical column with an integrated HF-etched nESI tip. Samples were loaded directly onto the column using an ACQUITY UPLC M-Class System (Waters) at $600 \mathrm{nLmin}^{-1}$ for 20 min with Buffer A (0.1\% formic acid (FA)) and eluted at $300 \mathrm{~nL} \mathrm{~min}{ }^{-1}$ using a gradient altering the concentration of Buffer B ( $99.9 \%$ acetonitrile, $0.1 \% \mathrm{FA}$ ) from 2 to $32 \% \mathrm{~B}$ over $60 \mathrm{~min}$, then from 32 to $40 \% \mathrm{~B}$ in the next $10 \mathrm{~min}$, then increased to $80 \% \mathrm{~B}$ over $8 \mathrm{~min}$ period, held at $100 \% \mathrm{~B}$ for $2 \mathrm{~min}$, and then dropped to $2 \% \mathrm{~B}$ for another $10 \mathrm{~min}$. Reverse-phase separated peptides were infused into a Q-Exactive (Thermo Scientific) mass spectrometer and data acquired using data-dependent acquisition. Two methods were used to identify putative glycopeptides. Method A aimed to enable robust peptide identification in which one full precursor scan (resolution 70,000; 350-1850 $/ 2$, AGC target of $1 \times 10^{6}$ ) was followed by 10 data-dependent higher energy collisional dissociation (HCD) MS-MS events (resolution 35k AGC target of $1 \times 10^{5}$ with a maximum injection time of $110 \mathrm{~ms}$, NCE 26 with $25 \%$ stepping) with $90 \mathrm{~s}$ dynamic exclusion enabled. Method B aimed to enable more complete characterization of glycans within glycopeptides with one full precursor scan (resolution 70,000; $350-1850 \mathrm{~m} / \mathrm{z}$, AGC target of $\left.1 \times 10^{6}\right)$ followed by 10 data-dependent HCD MS-MS events (resolution 35k AGC target of $5 \times 10^{5}$ with a maximum injection time of $250 \mathrm{~ms}$, NCE 13 with $25 \%$ stepping) with $90 \mathrm{~s}$ dynamic exclusion enabled.

Database interrogation of identified glycopeptides. Raw files were processed manually to identify potential glycopeptides based on the diagnostic oxonium $204.08 \mathrm{~m} / z$ ion. Putative glycopeptide-derived scans were manually inspected and identified as possible GluC-derived ComP glycopeptides based on the presence of an intense deglycosylated ComP-derived peptide ion, matching within $10 \mathrm{ppm}$ using the Expasy FindPept tool (https://web.expasy.org/findpept/). To facilitate peptide assignments, the resulting glycopeptides was manually annotated according to ref. ${ }^{46}$ with the aid of the Protein Prospector tool MS-Product (http://prospector. ucsf.edu/prospector/cgi-bin/msform.cgi?form $=$ msproduct).

Intact protein analysis. Intact analysis was performed using a 6520 Accurate Mass Quadrupole Time-of-Flight mass spectrometer (Agilent, Santa Clara, CA, USA). Protein samples were re-suspended in $2 \% \mathrm{ACN}, 0.1 \%$ trifluoroacetic acid, and immediately loaded onto a C5 Jupiter $5 \mu \mathrm{m} 300 \AA 50 \mathrm{~mm} \times 2.1 \mathrm{~mm}$ column (Phenomenex, Torrance, CA, USA) using an Agilent 1200. Samples were desalted by washing with buffer $\mathrm{A}(2 \% \mathrm{ACN}, 0.1 \% \mathrm{FA})$ for 4 min and then separated with a 12 min linear gradient from 2 to $100 \%$ buffer B $(80 \% \mathrm{ACN}, 0.1 \% \mathrm{FA})$ at a flow rate of $0.200 \mathrm{~mL} \mathrm{~min}^{-1}$. MS1 mass spectra were acquired at $1 \mathrm{~Hz}$ between a mass range of $300-3000 \mathrm{~m} / z$. Intact mass analysis and deconvolution was performed using MassHunter B.06.00 (Agilent).

Opsonophagocytosis assay. The assays were performed as previously described 47,48 and are briefly described below. Blood collection. Blood was collected by intracardiac puncture from naive female mice (Charles River, Wilmington, MA, USA), treated with sodium heparin, then diluted to obtain $6.25 \times 10^{6}$ leukocytes $\mathrm{mL}^{-1}$ in RPMI-1640 supplemented with $5 \%$ heat-inactivated fetal bovine serum, $10 \mathrm{mM}$ HEPES, $2 \mathrm{mM}$ L-glutamine, and $50 \mu \mathrm{M}$ 2-mercaptoethanol. All reagents were from Gibco (Invitrogen, Burlington, ON, Canada). Bacterial suspension preparation. Isolated colonies on sheep blood agar plates of either $S$. pneumoniae serotypes 8 or 14 (Statens Serum Institut, Denmark) were inoculated in $5 \mathrm{~mL}$ of Todd-Hewitt Broth (THB) (Oxoid, Thermo Fisher Scientific, Nepean, Canada) and incubated for $16 \mathrm{~h}$ at $37^{\circ} \mathrm{C}$ with $5 \% \mathrm{CO}_{2}$. Working cultures were prepared by transferring $0.1 \mathrm{~mL}$ of $16 \mathrm{~h}$ cultures into $10 \mathrm{~mL}$ of THB, which was then incubated for $5 \mathrm{~h}$. Bacteria were washed three times and re-suspended in PBS to obtain an $\mathrm{OD}_{600}$ value of 0.6 , which corresponds to $1.5 \times 10^{8}$ and colonyforming units (CFU) $\mathrm{mL}^{-1}$ and to $3.5 \times 10^{8} \mathrm{CFUmL}{ }^{-1}$ for serotype 8 and serotype 14, respectively. Final bacterial suspensions were prepared in complete cell culture medium to obtain a concentration of $6.25 \times 10^{4} \mathrm{CFUmL}^{-1}$. The number of $\mathrm{CFUmL}^{-1}$ in the final suspensions was determined by plating samples onto Todd-Hewitt agar (THA). Opsonophagocytosis Assay. Diluted whole blood $\left(5 \times 10^{5}\right.$ total leukocytes) was mixed with $5 \times 10^{3} \mathrm{CFU}$ of $S$. pneumoniae serotype 8 or 14 (multiplicity of infection of 0.01$)$ and $5 \%\left(\mathrm{v} \mathrm{v}^{-1}\right)$ of serum from control (placebo) or vaccinated mice in a microtube to a final volume of $0.2 \mathrm{~mL}$. Microtubes were incubated for $4 \mathrm{~h}$ at $37^{\circ} \mathrm{C}$ with $5 \% \mathrm{CO}_{2}$, with shaking. After incubation, viable bacterial counts were performed on THA. Tubes with the addition of naive mouse sera or commercial rabbit anti-S. pneumoniae types 8 or 14 serum (Statens Serum Institut, Denmark) were used as negative and positive controls, respectively. The percentage of bacterial killing was determined using the following formula: percent bacteria killed $=[1-$ (bacteria recovered from sample tubes/bacteria recovered from negative control tubes with naive sera) $] \times 100$.

Reporting summary. Further information on experimental design is available in the Nature Research Reporting Summary linked to this article.

\section{Data availability}

The authors declare that data supporting the findings of this study are available within the paper and its supplemental files. The source data underlying Figs. 1, 3, 4, 5, 6, 7 and supplemental Figs. 2 and 5 are provided as a Source Data file.

Received: 7 August 2018 Accepted: 5 February 2019

Published online: 21 February 2019

\section{References}

1. Hausdorff, W. P., Hoet, B. \& Adegbola, R. A. Predicting the impact of new pneumococcal conjugate vaccines: serotype composition is not enough. Expert. Rev. Vaccin. 14, 413-428 (2015).

2. O’Brien, K. L. et al. Burden of disease caused by Streptococcus pneumoniae in children younger than 5 years: global estimates. Lancet 374, 893-902 (2009).

3. WHO. Estimated Hib and Pneumococcal Deaths for Children under 5 years of Age, 2008. http://www.who.int/immunization/monitoring_surveillance/ burden/estimates/Pneumo_hib/en (2013).

4. CDC. Pneumococcal Disease. https://www.cdc.gov/pneumococcal/vaccination. html (2017).

5. Cadoz, M. Potential and limitations of polysaccharide vaccines in infancy. Vaccine 16, 1391-1395 (1998)

6. Avci, F. Y., Li, X., Tsuji, M. \& Kasper, D. L. A mechanism for glycoconjugate vaccine activation of the adaptive immune system and its implications for vaccine design. Nat. Med. 17, 1602-1609 (2011).

7. Pollard, A. J., Perrett, K. P. \& Beverley, P. C. Maintaining protection against invasive bacteria with protein-polysaccharide conjugate vaccines. Nat. Rev. Immunol. 9, 213-220 (2009).

8. CDC. Vaccines for Children Program (VFC). https://www.cdc.gov/vaccines/ programs/vfc/awardees/vaccine-management/price-list/index.html (2018).

9. Pfizer. Pfizer Inc. 2017 Financial Report. https://www.sec.gov/Archives/edgar/ data/78003/000007800318000027/pfe-exhibit13x12312017x10k.htm (2017).

10. Wahl, B. et al. Burden of Streptococcus pneumoniae and Haemophilus influenzae type b disease in children in the era of conjugate vaccines: global, regional, and national estimates for 2000-15. Lancet Glob. Health 6 , e744-e757 (2018).

11. Loo, J. D. et al. Systematic review of the effect of pneumococcal conjugate vaccine dosing schedules on prevention of pneumonia. Pediatr. Infect. Dis. J. 33, S140-S151 (2014)

12. Grabenstein, J. D. \& Musey, L. K. Differences in serious clinical outcomes of infection caused by specific pneumococcal serotypes among adults. Vaccine 32, 2399-2405 (2014).

13. Cui, Y. A., Patel, H., O’Neil, W. M., Li, S. \& Saddier, P. Pneumococcal serotype distribution: a snapshot of recent data in pediatric and adult populations around the world. Hum. Vaccin. Immunother. 13, 1-13 (2017).

14. Gladstone, R. A. et al. Five winters of pneumococcal serotype replacement in UK carriage following PCV introduction. Vaccine 33, 2015-2021 (2015).

15. Frasch, C. E. Preparation of bacterial polysaccharide-protein conjugates: analytical and manufacturing challenges. Vaccine 27, 6468-6470 (2009). 
16. Feldman, M. F. et al. Engineering $N$-linked protein glycosylation with diverse $\mathrm{O}$ antigen lipopolysaccharide structures in Escherichia coli. Proc. Natl. Acad. Sci. USA 102, 3016-3021 (2005).

17. Huttner, A. et al. Safety, immunogenicity, and preliminary clinical efficacy of a vaccine against extraintestinal pathogenic Escherichia coli in women with a history of recurrent urinary tract infection: a randomised, single-blind, placebo-controlled phase 1b trial. Lancet Infect. Dis. 17, 528-537 (2017).

18. Riddle, M. S. et al. Safety and immunogenicity of a candidate bioconjugate vaccine against Shigella flexneri 2a administered to healthy adults: a singleblind, randomized phase I study. Clin. Vaccin. Immunol. 23, 908-917 (2016).

19. Nothaft, H. \& Szymanski, C. M. Protein glycosylation in bacteria: sweeter than ever. Nat. Rev. Microbiol. 8, 765-778 (2010).

20. Iwashkiw, J. A., Vozza, N. F., Kinsella, R. L. \& Feldman, M. F. Pour some sugar on it: the expanding world of bacterial protein $\mathrm{O}$-linked glycosylation. $\mathrm{Mol}$. Microbiol. 89, 14-28 (2013).

21. Geno, K. A. et al. Pneumococcal capsules and their types: past, present, and future. Clin. Microbiol. Rev. 28, 871-899 (2015).

22. Iwashkiw, J. A. et al. Exploiting the Campylobacter jejuni protein glycosylation system for glycoengineering vaccines and diagnostic tools directed against brucellosis. Microb. Cell Fact. 11, 13 (2012).

23. Ciocchini, A. E. et al. Development and validation of a novel diagnostic test for human brucellosis using a glyco-engineered antigen coupled to magnetic beads. PLoS Negl. Trop. Dis. 7, e2048 (2013).

24. Wacker, M. et al. Substrate specificity of bacterial oligosaccharyltransferase suggests a common transfer mechanism for the bacterial and eukaryotic systems. Proc. Natl. Acad. Sci. USA 103, 7088-7093 (2006).

25. Faridmoayer, A. et al. Extreme substrate promiscuity of the Neisseria oligosaccharyl transferase involved in protein O-glycosylation. J. Biol. Chem. 283, 34596-34604 (2008)

26. Faridmoayer, A., Fentabil, M. A., Mills, D. C., Klassen, J. S. \& Feldman, M. F. Functional characterization of bacterial oligosaccharyltransferases involved in O-linked protein glycosylation. J. Bacteriol. 189, 8088-8098 (2007).

27. Bentley, S. D. et al. Genetic analysis of the capsular biosynthetic locus from all 90 pneumococcal serotypes. PLoS Genet. 2, e31 (2006).

28. Harding, C. M. et al. Acinetobacter strains carry two functional oligosaccharyltransferases, one devoted exclusively to type IV pilin, and the other one dedicated to O-glycosylation of multiple proteins. Mol. Microbiol. 96, 1023-1041 (2015).

29. Ihssen, J. et al. Increased efficiency of Campylobacter jejuni $\mathrm{N}$ oligosaccharyltransferase PglB by structure-guided engineering. Open Biol. 5, 140227 (2015)

30. Kowarik, M. et al. Definition of the bacterial $N$-glycosylation site consensus sequence. EMBO J. 25, 1957-1966 (2006).

31. Vik, A. et al. Broad spectrum $O$-linked protein glycosylation in the human pathogen Neisseria gonorrhoeae. Proc. Natl. Acad. Sci. USA 106, 4447-4452 (2009).

32. Scott, N. E. et al. Diversity within the $O$-linked protein glycosylation systems of Acinetobacter species. Mol. Cell. Proteom. 13, 2354-2370 (2014).

33. Comer, J. E., Marshall, M. A., Blanch, V. J., Deal, C. D. \& Castric, P. Identification of the Pseudomonas aeruginosa 1244 pilin glycosylation site. Infect. Immun. 70, 2837-2845 (2002).

34. Wuorimaa, T. et al. Avidity and subclasses of IgG after immunization of infants with an 11-valent pneumococcal conjugate vaccine with or without aluminum adjuvant. J. Infect. Dis. 184, 1211-1215 (2001).

35. Soininen, A., Seppala, I., Nieminen, T., Eskola, J. \& Kayhty, H. IgG subclass distribution of antibodies after vaccination of adults with pneumococcal conjugate vaccines. Vaccine 17, 1889-1897 (1999).

36. Pilishvili, T. et al. Sustained reductions in invasive pneumococcal disease in the era of conjugate vaccine. J. Infect. Dis. 201, 32-41 (2010).

37. Herbert, J. A. et al. Production and efficacy of a low-cost recombinant pneumococcal protein polysaccharide conjugate vaccine. Vaccine 36, 3809-3819 (2018).

38. Pan, C. et al. Biosynthesis of conjugate vaccines using an $\mathrm{O}$-linked glycosylation system. mBio 7, e00443-00416 (2016).

39. Sun, P. et al. Design and production of conjugate vaccines against $S$. paratyphi A using an $O$-linked glycosylation system in vivo. NPJ Vaccin. 3, 4 (2018).

40. Castric, P. pilO, a gene required for glycosylation of Pseudomonas aeruginosa 1244 pilin. Microbiology 141, 1247-1254 (1995).

41. Aas, F. E., Vik, A., Vedde, J., Koomey, M. \& Egge-Jacobsen, W. Neisseria gonorrhoeae O-linked pilin glycosylation: functional analyses define both the biosynthetic pathway and glycan structure. Mol. Microbiol. 65, 607-624 (2007).

42. Aas, F. E. et al. Neisseria gonorrhoeae type IV pili undergo multisite, hierarchical modifications with phosphoethanolamine and phosphocholine requiring an enzyme structurally related to lipopolysaccharide phosphoethanolamine transferases. J. Biol. Chem. 281, 27712-27723 (2006).
43. Plotkin, S. A. Correlates of protection induced by vaccination. Clin. Vaccin. Immunol. 17, 1055-1065 (2010).

44. Ishihama, Y., Rappsilber, J. \& Mann, M. Modular stop and go extraction tips with stacked disks for parallel and multidimensional peptide fractionation in proteomics. J. Proteome Res. 5, 988-994 (2006).

45. Rappsilber, J., Mann, M. \& Ishihama, Y. Protocol for micro-purification, enrichment, pre-fractionation and storage of peptides for proteomics using StageTips. Nat. Protoc. 2, 1896-1906 (2007).

46. Roepstorff, P. \& Fohlman, J. Proposal for a common nomenclature for sequence ions in mass spectra of peptides. Biomed. Mass Spectrom. 11, 601 (1984).

47. Goyette-Desjardins, G., Roy, R. \& Segura, M. Murine whole-blood opsonophagocytosis assay to evaluate protection by antibodies raised against encapsulated extracellular bacteria. Methods Mol. Biol. 1331, 81-92 (2015).

48. Price, N. L. et al. Glycoengineered outer membrane vesicles: a novel platform for bacterial vaccines. Sci. Rep. 6, 24931 (2016).

49. Kay, E. J., Yates, L. E., Terra, V. S., Cuccui, J. \& Wren, B. W. Recombinant expression of Streptococcus pneumoniae capsular polysaccharides in Escherichia coli. Open Biol. 6, 150243 (2016).

\section{Acknowledgements}

We would like to thank Brendan Wren and Laura Yates for providing the $\mathrm{pB}-8$ plas$\mathrm{mid}^{49}$. We would like to thank Andrew Webb from Walter Eliza Hall institute of medical research for providing mass spectrometry support for the peptide-based analysis. We would also like to thank the Melbourne Mass Spectrometry and Proteomics Facility of The Bio21 Molecular Science and Biotechnology Institute at The University of Melbourne for the support, maintenance, and access to mass spectrometry infrastructure for intact protein-based analysis. This work was funded by National Institute for Allergy and Infectious Disease (NIAID) R41 AI131742 grant awarded to M.F.F. and VaxNewMo. This work was partially supported by National Health and Medical Research Council of Australia (NHMRC) project grants awarded to N.E.S. (APP1100164). N.E.S. was supported by an Overseas (Biomedical) Fellowship (APP1037373) and a University of Melbourne Early Career Researcher Grant Scheme (Proposal number 603107). J.P.H. was supported by a National Science Foundation Graduate Research Fellowship (DGE1143954), R.L.K. was supported by a Potts Memorial Foundation Postdoctoral Fellowship, and C.L.S. was supported by a Burroughs Wellcome Fund Investigators in the Pathogenesis of Infectious Disease award.

\section{Author contributions}

C.M.H., M.A.N., N.E.S., G.G.-D., H.N., A.E.M., S.M.C., J.P.H., R.L.K., all performed experiments related to this study. M.A.N., C.M.H., N.E.S. and M.F.F. analyzed data sets. C.M.S., C.L.S., M.S. and M.F.F. directed the study. C.M.H., M.A.N., and M.F.F. wrote the manuscript

\section{Additional information}

Supplementary Information accompanies this paper at https://doi.org/10.1038/s41467 019-08869-9.

Competing interests: The authors declare no competing interests.

Reprints and permission information is available online at http://npg.nature.com/ reprintsandpermissions/

Journal peer review information: Nature Communications thanks the anonymous reviewers for their contribution to the peer review of this work. Peer reviewer reports are available.

Publisher's note: Springer Nature remains neutral with regard to jurisdictional claims in published maps and institutional affiliations.

Open Access This article is licensed under a Creative Commons Attribution 4.0 International License, which permits use, sharing, adaptation, distribution and reproduction in any medium or format, as long as you give appropriate credit to the original author(s) and the source, provide a link to the Creative Commons license, and indicate if changes were made. The images or other third party material in this article are included in the article's Creative Commons license, unless indicated otherwise in a credit line to the material. If material is not included in the article's Creative Commons license and your intended use is not permitted by statutory regulation or exceeds the permitted use, you will need to obtain permission directly from the copyright holder. To view a copy of this license, visit http://creativecommons.org/ licenses/by/4.0/

(C) The Author(s) 2019 